\title{
Sensing Cloud Optimization to Solve ED of Units with Valve-Point Effects and Multi-fuels
}

\author{
Pedro Fonte ${ }^{1,2}$, Claudio Monteiro ${ }^{2}$, and Fernando Maciel Barbosa ${ }^{2,3}$ \\ ${ }^{1}$ ISEL - Lisbon Superior Engineering Institute, Lisbon, Portugal \\ pfonteldeea.isel.pt \\ ${ }^{2}$ University of Porto \\ ${ }^{3}$ INESC TEC PORTO, Porto, Portugal \\ $\{\mathrm{cdm}, \mathrm{fmb}\} @$ fe.up.pt
}

\begin{abstract}
In this paper a solution to an highly constrained and non-convex economical dispatch (ED) problem with a meta-heuristic technique named Sensing Cloud Optimization (SCO) is presented. The proposed meta-heuristic is based on a cloud of particles whose central point represents the objective function value and the remaining particles act as sensors "to fill" the search space and "guide" the central particle so it moves into the best direction. To demonstrate its performance, a case study with multi-fuel units and valve- point effects is presented.
\end{abstract}

Keywords: Economic dispatch, Optimization, Heuristics, Cloud of particles.

\section{Introduction}

The Economical Dispatch (ED) problem is an important issue in the power system operation. Fundamentally it is intended to evaluate the value that each on-line unit should generate with the lowest cost, as (1), respecting the technical and load demand constrains. The ED uses as a basis the Unit Commitment (UC) solution, excluding from the optimization the generation units that are off. It is common to adopt approaches that merge the problem of UC with the problem of ED using Integer Mixed Programming optimization algorithms. This can have advantages because the non-linear detail in the ED can justify a change in the solution of the UC. On the other hand, the ED can integrate UC costs considering the fix costs of the generation [1].

$$
\min \sum_{i=1}^{N_{G}} F_{i}\left(P_{i}\right)
$$

Where $F_{i}$ is the cost function of each unit $P_{i}$, and $N_{G}$ represents the number of on-line units [2]. Over the past decade, many methods have been developed to solve the economical dispatch problem. There are traditional methods such as Gradient, Lagrangean function, Lambda-iteration method, Dynamic Programming, Newton's method, Linear Programming and Interior Point method, among others [3]. However, the generation cost functions of recent thermal units are not continues, not convex, neither differentiable due to valve-point loading effect, multi-fuel burn systems and 
operational prohibited zones. Thus, the ED problem becomes a non-convex optimization problem with constrains, which cannot be solved directly by some of the traditional mathematical methods. Generally, dynamic programming can solve this kind of problems, but can suffer with the dimension and time needed to solve it [4-6]. Due to that several heuristic methods were proposed to solve this kind of problems such as Genetic Algorithms (GA), Simulated Annealing (SA), Taboo Search (TS), Evolutionary Programming (EP), Evolutionary Strategies (ES), Particle Swarm Optimization (PSO), Artificial Neural Networks approach with Hopfield Networks and hybrid artificial intelligence methods [7]. From the base algorithms several improved approaches and hybrid were proposed, as Improved Taboo Search [4], Fast Evolutionary Programming (FEP) and Improved Fast Evolutionary Programming (IFEP)[8], Improved Particle Swarm Optimization (IPSO)[7], PSO embedded Evolutionary Programming (PSO-EP) [9], PSO with crossover operations [7] and Fast Evolutionary programming with Swarm Direction [2]. Other techniques as Bacteria Foraging Optimization (BFO), Ant Colony Optimization (ACO) [3][11], Combination of Chaotic Differential Evolution and Quadratic Programming [12], a special class of ant colony (API) and Real Coded Genetic Algorithm (GA-API) can be considered as well [13] or Improved Genetic Algorithm with Multiplier Updating (IGA_MU) and Conventional Genetic Algorithm with Multiplier Updating (CGA_MU)[10], among many others. In this paper is proposed to solve an ED problem of units with multifuels and valve-point effects using the algorithm called Sensing Cloud Optimization (SCO)[14]. It is a stochastic technique based in a cloud of particles with parallel search without presenting evolutionary behaviors. There is no competition between the particles or self-adaptation of their characteristics being a purely cooperative system, since all contribute to reach the optimum value.

The heuristics techniques due to its stochastic behavior can be trapped in local minima; therefore, the added value of SCO is the dynamic mechanism to avoid, as better as possible, to be trapped in local minima [14]. From the work developed up to now, it has demonstrated precise results as well as the capacity to deal with large quantity of variables [16]. In this paper is intended to continue the research solving an ED of units with valve-point effects and multi-fuels. In future works we intend to develop an integrated set of mathematic techniques to optimise the operation of electric energy systems with significant penetration of high variability resources. The final result should be a complete methodological suit idealised to answer integrally to the dispatch of energy systems with high integration of variable power resources.

\section{Contribution to Internet of the Things}

The Economic Dispatch, Security Constrained Economic Dispatch (SCED) and Reliability Constraint Economic Dispatch (RCED) are some of the most important optimization problems in a power system scheduling. The result is the mix of thermal power plants production under technical and security constraints at the lowest price. The optimization of the electricity cost makes the society and economy more sustainable and competitive. In an Internet of the things perspective, the power units connected to the internet gain the capacity to communicate and share information 
among them. As well as, the capacity to configure and decide autonomously, when and how much produce, under an optimal perspective, optimizing the production cost and environmental impacts. This share of information allows technical decisions, maintaining the security of electrical energy supply. Beyond that, in a global perspective allows the scheduling of the decision chain since the extraction of fossil fuels, its transportation, distribution and utilization. The introduction of SCO algorithm aims to provide a tool with good precision to solve high dimensional and constrained ED problems.

\section{Problem Description}

Traditional ED problems characterise the cost function as quadratic, but in more recent thermal units, an absolute term is added to the quadratic cost function due to valve point effects. Beyond this, some thermal units, as combined cycle, operate with different kind of fuels and multiple cost curves, resulting an "hybrid cost function" as (2), represented with several piecewise functions reflecting the effects of fuel type $k$ [10]. Where $a_{i}, b_{i}, c_{i}, e_{i}$ and $f_{i}$ are fuel cost coefficients of the $i^{\text {th }}$ unit, and $P_{i}$ and $P_{i}^{\mathrm{min}}$ represent the production and minimum limit of $i^{\text {th }}$ unit, respectively [12].

$$
F_{i}\left(P_{i}\right)= \begin{cases}a_{i 1}+b_{i 1} P_{i}+c_{i 1} P_{i}^{2}+\left|e_{i 1} \times \sin \left(f_{i 1} \times\left(P_{i 1}^{\min }-P_{i 1}\right)\right)\right|, & \text { for fuel } 1, P_{i 1}^{\min } \leq P_{i} \leq P_{i 1} \\ a_{i 2}+b_{i 2} P_{i}+c_{i 2} P_{i}^{2}+\left|e_{i 2} \times \sin \left(f_{i 2} \times\left(P_{i 2}^{\min }-P_{i 2}\right)\right)\right|, & \text { for fuel } 2, P_{i 1}^{\min }<P_{i} \leq P_{i 2} \\ \vdots & \\ a_{i k}+b_{i k} P_{i}+c_{i k} P_{i}^{2}+\left|e_{i k} \times \sin \left(f_{i k} \times\left(P_{i k}^{\min }-P_{i k}\right)\right)\right|, & \text { for fuel } k, P_{i k-1}<P_{i} \leq P_{i}^{\max }\end{cases}
$$

In an Electric Power System, the total power production must equal the load demand $D$ and power transmission losses $P_{L}$, as (3). The power losses are function of unit's power outputs and can be calculated by the losses coefficients matrix $\mathbf{B}$ [13].

$$
\sum_{i=1}^{N_{G}} P_{i}=P_{L}+D
$$

Simultaneously several technical operation constrains must be satisfied such as minimum and maximum power generation and ramp limits. The output of each unit must be within its maximum $P_{i}^{\max }$ and minimum limits $P_{i}^{\text {min }}$. The increasing ramp limit is expressed by $U R_{i}$ and $D R_{i}$ represents the decreasing ramp limit. Together with minimum and maximum limits, results (4), where $P_{i}$ represents the power output of $i^{\text {th }}$ unit at a given time interval, and $P_{i}^{o}$ the power at previous time interval,

$$
\max \left(P_{i}^{\min }, P_{i}^{0}-D R_{i}\right) \leq P_{i} \leq \min \left(P_{i}^{\max }, P_{i}^{0}+U R_{i}\right) .
$$

In some cases, the total operation range of a generating unit is not always available due to physical operation limitations, as steam valve operation, vibrations in the shaft 
bearings, among others, resulting in prohibited operating zones. In practical operation, the adjustment of power output $P_{i}$ must avoid operating in the prohibited zones.

\section{$4 \quad$ Algorithm}

In [14] and [16], SCO showed appropriate heuristic characteristic to solve not convex, not differentiable and highly constrained optimisation problems. It is characterized by two distinct steps, the cloud particles fitness evaluation and a statistical analysis to determine the cloud's direction and dimension. The cloud's dimension presents a dynamic adjustment in search space in order to accelerate the convergence and to avoid to get trapped in local minima. It was introduced the concept of central particle, which tries to find the optimal value, being the remaining particles of the cloud spread around, according with a Gaussian distribution. By this way, the search space covered by the cloud can be dynamically controlled only by the variance of the distribution. These particles will act as sensors "to fill" the search space and give "signals" to the central particle moves into the best direction in the search space. The adaptive adjustment of the cloud's dimension is performed by two inverted sigmoid. The algorithm when applied to the ED problem can be described by the following 10 steps.

Step 1. Create randomly a central particle $P_{q(i)}$, with $i=1 . . N_{G}$ dimensions, representing each generation unit according to its limits, as in (5) (If there are starting values, $\left.P_{q(i)}=P_{(i)}^{o}\right)$,

$$
P_{q(i)}=\operatorname{rand}(0,1) \cdot\left(P_{i}^{\max }-P_{i}^{\min }\right)+P_{i}^{\min }
$$

Create the remaining cloud $\left[N_{G} \times N_{P}\right]$ with $j=1 . . N_{P}$ particles and $i=1 . . N_{G}$ dimensions normally distributed centered in the central particle and standard deviation $\sigma_{(i)}^{(k=1)}$.

Step 2. To each cloud particle $P_{(j)}^{(k)}$, calculate the transmission loss and evaluate each particle with (6) and retain the best fitness value $P_{(j) B E S T}^{(k)}$ and its position.

$$
f_{(j)}^{(k)}=\sum_{i=1}^{N_{G}} F_{i}\left(P_{(i, j)}^{(k)}\right)+\left(\sum_{i=1}^{N_{G}} P_{(i, j)}^{(k)}-D-P_{L}^{(k)}\right)^{2}
$$

In this work, to the traditional fitness function (1), a quadratic penalty has added to decrease the deviation between the power production and the sum of power demand and active losses.

Step 3. Calculate the second order regression coefficients to each dimension $i$, $\left[\beta_{0(i)} \beta_{1(i)} \beta_{2(i)}\right]$ and the determination coefficient $R_{q(i)}^{2(k)}$.

Step 4. Verify the convexity of polynomials. Depending of the function to be optimized and the search space covered by the cloud, the second-order fitness function can be concave indicating a maximum instead a minimum. In this case is 
necessary to calculate the trend point in order to ensure that the central point continues to move toward a minimum. This is done calculating the roots of the concave function subtracted by the best fitness, as indicated in (7) and choose the root closest of $P_{(j) B E S T}^{(k)}$ by (8);

$$
\begin{aligned}
& \beta_{0(i)}+\beta_{1(i)} P_{(i)}^{(k)}+\beta_{2(i)} P_{(i)}^{2(k)}-f_{(j)}^{(k)}\left(P_{(i) B E S T}^{(k)}\right)=0 \\
& t_{p(i)}^{(k)}=\min \left\{\left|P_{(i) 1}^{(k)}-P_{(i) B E S T}^{(k)}\right|,\left|P_{(i) 2}^{(k)}-P_{(i) B E S T}^{(k)}\right|\right\} .
\end{aligned}
$$

Otherwise, if it is convex, $t_{p(i)}^{(k)}=-b_{i} / 2 a_{i}$.

Step 5. Generate new central particle by (9) and verify if satisfies all constrains.

$$
P_{q(i)}^{(k+1)}=t_{p(i)}^{(k)} \cdot R_{q(i)}^{2}+\left(1-R_{q(i)}^{2}\right) \cdot P_{(i) B E S T}^{(k)} .
$$

If the central particle satisfies all constraints, then it is a feasible solution. (As remain particles acts as sensors and are not candidates to optimal solution they don't need to satisfy all constrains).

Step 6. Calculate the Euclidean distance for each particle $j$ to the central particle, by (10)

$$
d_{(j)}^{(k)}=\left\|P_{q}^{(k)}-P_{(j)}^{(k)}\right\|
$$

Step 7. Calculate the linear regression coefficients $\left[\alpha_{o(j)}, \alpha_{1(j)}\right]$ of $P_{(j)}^{(k)}=f\left(d_{(j)}^{(k)}\right)$ and determinate the coefficient $R_{\varphi(j)}^{2(k)}$.

Step 8. Calculate the new standard deviation for each dimension $i$ of new cloud by (11)

$$
\sigma_{(i)}^{2(k+1)}=\sigma_{(i)}^{2(k)} \cdot F_{s 1(j)} \cdot F_{s 2(i, j)} \cdot
$$

The changing in cloud variance and, consequently, in the cloud dimension is done by two inverted sigmoid (12) and (13). In (12), $\Delta \varphi$ is calculated by (14) and (15).

$$
\begin{gathered}
F_{s 1(k)}=1+\frac{\Delta \varphi-1}{e^{\left(\frac{-8(t-t c)}{t s}\right)}} \\
F_{s 2(i, k)}=1-\frac{(K-1)}{1+e^{\left(-m R_{s(i)}^{2}+n\right)}} \\
\nabla \varphi=\frac{d f\left(\varphi_{(j)}\right)}{d\left(\varphi_{(j)}\right)}\left(\sigma_{\varphi(j)} / \sigma_{\text {fitness }(j)}\right)
\end{gathered}
$$




$$
\Delta \varphi=\frac{1}{1+h . \nabla \varphi}
$$

Step 9. If $k=i t_{\max }$ go to step 10 , otherwise, $k=k+1$ and go to step 1 .

Step 10. The central particle that generates the minimum value is the optimal generation power of each unit.

\section{$5 \quad$ Numerical Examples and Results}

One case is studied to verify the capacity to solve non-convex and constrained problems and reach the optimal values. As heuristic methods may not converge exactly to the same solution each run owing to their stochastic behaviour, their performances could not be judged by the results of a single trial. Due to that, the case study will be performed 50 times. In power systems literature, generally, the convergence is mainly related with number of iterations or generations [2], [6], [8-10] or CPU time per iteration /generation [4]. However, this way doesn't give adequate information about the computational effort to perform a task in order to have the same base of comparison [13]. Thus, in this paper to compare the computational efforts independently of the CPU or number of iterations, the numbers of objective and constraints functions evaluations are used.

\subsection{Case Study}

The test system consists of 10 thermal units with valve-point effects and multi-fuels without forbidden operation zones or ramp limits, neither power losses, feeding a load demand of $2700 \mathrm{MW}$. All required data is available in [10]. To solve this case, the central particle as well as, the remaining particles of the cloud will have 10 dimensions $\left(P_{1} \ldots P_{10}\right)$, one for each generation unit. Depending on the number of particles $N_{P}$, the dimension of cloud is [10 x $N_{P}$ ]. The number of iterations was limited to 200. As referred in [14] the number of particles has not excessive importance because there isn't a direct relation between the increase of its number and the increase of the performance. On the other hand, as they act as "sensors" in the search space and are used to calculate the first and second order polynomials, there is a minimum number necessary to describe the curve fitting. Due to this, after some experiences the particles number was set to 10 . In (15) $h$ was set to 1 , in (12) $t_{c}$ was set to 0,5 and $t_{s}$ to 1 and in (13) the values of $K, m$ and $n$ were set, respectively to $1.02,50$ and 5 .

As indicated in [10], each unit has different cost functions and operation limits depending on the fuel. The results will be the cost value obtained by the minimization of (7) subject to (4). The problem includes one objective function with 10 variables and 20 inequality constraints. In figure 1 is shown the convergence behavior being the best value reached after 1800 cost function evaluations. 


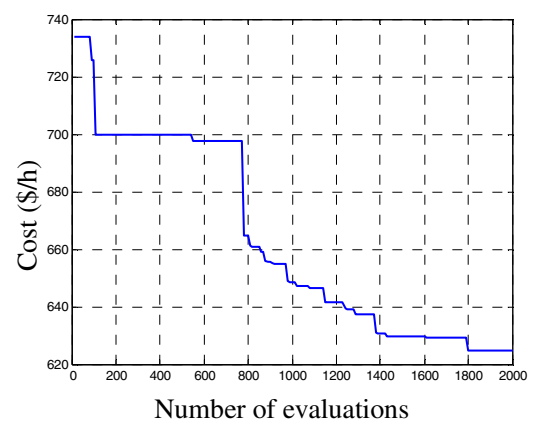

Fig. 1. Convergence behaviours of SCO for a 10-units problem

The results reached by SCO were compared with CGA_MU and IGA_MU[10] as well as with PSO-LRS, NPSO and NPSO_LRS[6], the results are shown in table 1. It can be concluded that all the proposed methods reach the same combination of fuels and SCO reached comparable values with other proposed metaheuristics.

Table 1. Results obtained (10-unis 2700 MW) (Best individual)

\begin{tabular}{|c|c|c|c|c|c|c|c|}
\hline \multirow{2}{*}{$\begin{array}{l}\text { Power } \\
(M W)\end{array}$} & \multicolumn{7}{|c|}{ Method } \\
\hline & $\mathrm{SCO}$ & $C G A \_M U$ & $I G A \_M U$ & PSO-LRS & NPSO & NPSO-LRS & fuel \\
\hline $\mathrm{P}_{\mathrm{G} 1}$ & 225,612 & 222,0108 & 219,1261 & 219,1261 & 220,6570 & 223,3352 & 2 \\
\hline $\mathrm{P}_{\mathrm{G} 2}$ & 207,103 & 211,6352 & 211,1645 & 211,1645 & 211,7859 & 212,1957 & 1 \\
\hline $\mathrm{P}_{\mathrm{G} 3}$ & 281,576 & 283,9455 & 280,6572 & 280,6572 & 280,4026 & 276,2167 & 1 \\
\hline $\mathrm{P}_{\mathrm{G} 4}$ & 237,912 & 237,8052 & 238,4770 & 238,4770 & 238,6013 & 239,4187 & 3 \\
\hline $\mathrm{P}_{\mathrm{G} 5}$ & 271,667 & 280,4480 & 276,4179 & 276,4179 & 277,5621 & 274,6470 & 1 \\
\hline $\mathrm{P}_{\mathrm{G} 6}$ & 242,476 & 236,0330 & 240,4672 & 240,4672 & 239,1204 & 239,7974 & 3 \\
\hline $\mathrm{P}_{\mathrm{G} 7}$ & 284,633 & 292,0499 & 287,7399 & 287,7399 & 292,1397 & 285,5388 & 1 \\
\hline $\mathrm{P}_{\mathrm{G} 8}$ & 243,321 & 241,9708 & 240,7614 & $240,76,14$ & 239,1530 & 240,6323 & 3 \\
\hline $\mathrm{P}_{\mathrm{G} 9}$ & 432,693 & 424,2011 & 429,3370 & 429,3370 & 426,1142 & 429,2637 & 3 \\
\hline $\mathrm{P}_{\mathrm{G} 10}$ & 273,006 & 269,9005 & 275,8518 & 275,8518 & 274,4637 & 278,9541 & 1 \\
\hline $\mathrm{P}_{\mathrm{T}}(\mathrm{MW})$ & 2700 & 2700 & 2700 & 2700 & 2700 & 2700 & \\
\hline Cost $(\$ / h)$ & 624,65 & 624,72 & 624,52 & 624,23 & 624,16 & 624,13 & \\
\hline Evaluat. & 1800 & N/A & N/A & 3440 & 3240 & 2120 & \\
\hline
\end{tabular}

\section{Conclusions and Future Works}

This paper investigated the capability of SCO to solve highly non-convex and constrained ED problems as the case of units with valve-point effects and multi-fuels. The values reached are comparable with evolutionary methods as PSO and GA and other hybrid solutions. The compared algorithms are hybrid models or evolution from base algorithms. SCO is the first approach which in future could be improved and achieve better performances. 


\section{References}

1. Catalão, J.P.S.: Electric Power Systems: Advanced Forecasting Techniques and Optimal Generation Scheduling. CRC Press (2012)

2. Gaing, Z.-L., Ting-Chia, O.: Dynamic Economic Dispatch Solution Using Fast Evolutionary Programming with Swarm Direction. In: ICIEA 2009 - 4th IEEE Conference on Industrial Electronics and Applications, vol. (1), pp. 1538-1544 (2009)

3. Hazra, J., Sinha, A.: Application of soft computing methods for Economic Dispatch in Power Systems. International Journal of Electrical and Electronics Engineering (2), 538-543 (2009)

4. Gaing, Z.-L.: Particle swarm optimization to solving the economic dispatch considering the generator constraints. IEEE Transactions on Power Systems 18(3), 1187-1195 (2003)

5. Lin, W.-M., Cheng, F.-S., Tsay, M.-T.: An improved tabu search for economic dispatch with multiple minima. IEEE Transactions on Power Systems 17(1), 108-112 (2002)

6. Selvakumar, A.I., Thanushkodi, K.: A New Particle Swarm Optimization Solution to Nonconvex Economic Dispatch Problems. IEEE Transactions on Power Systems 22(1), 42-51 (2007)

7. Lee, K.Y.: An Improved Particle Swarm Optimization for Nonconvex Economic Dispatch Problems. IEEE Transactions on Power Systems 25(1), 156-166 (2010)

8. Sinha, N., Chakrabarti, R., Chattopadhyay, P.K.: Evolutionary programming techniques for economic load dispatch. IEEE Transactions on Evolutionary Computation 7(1), 83-89 (2003)

9. Sinha, N., Purkayastha, B.: PSO embedded evolutionary programming technique for nonconvex economic load dispatch. In: IEEE PES Power Systems Conference and Exposition, vol. 1, pp. 66-71 (2004)

10. Chiang, C.-L.: Improved Genetic Algorithm for Power Economic Dispatch of Units With Valve-Point Effects and Multiple Fuels. IEEE Transactions on Power Systems 20(4), 1690-1699 (2005)

11. Serapião, A.: Fundamental of Optimization by Swarm Inteligence: an Overview, Fundamentos de Otimização por Inteligência de Enxames: uma Visão Geral. Revista Contole \& Automação 20(3) (July-September 2009) (in Portuguese)

12. Coelho, S., Mariani, V.C.: Combining of Chaotic Differential Evolution and Quadratic Programming for Economic Dispatch Optimization With Valve-Point Effect. IEEE Transactions on Power Systems 21(2), 989-996 (2006)

13. Ciornei, I., Kyriakides, E.: A GA-API Solution for the Economic Dispatch of Generation in Power System Operation. IEEE Transactions on Power Systems 27(1), 233-242 (2012)

14. Fonte, P.M., Monteiro, C., Barbosa, F.M.: SCO - Sensing Cloud Optimization - A Novel Optimization Technique (in reviewing)

15. Park, J.-B., Lee, K.-S., Shin, J.-R., Lee, K.Y.: A Particle Swarm Optimization for Economic Dispatch With Nonsmooth Cost Functions. IEEE Transactions on Power Systems 20(1), 34-42 (2005)

16. Fonte, P., Monteiro, C., Barbosa, F.M.: SCO - Sensing Cloud Optimization Applied to a Non-convex and Constrained Dynamic Economical Dispatch. IEEE Transactions on Power Systems (in reviewing) 